\title{
Delay Tolerant Network Simulation with VNUML
}

\author{
Lídice Romero Amondaray \\ Facultad de Ingeniería Eléctrica \\ Universidad de Oriente \\ Santiago de Cuba, 90900, Cuba \\ lidice@fie.uo.edu.cu
}

\author{
Joaquín Seoane Pascual \\ E.T.S.I.T Telecomunicación \\ Universidad Politécnica de Madrid \\ Ciudad Universitaria Madrid, E-28040, España \\ jsp@dit.upm.es
}

\begin{abstract}
This paper describes a simulation environment to easily create complex Delay Tolerant Network (DTN) scenarios using Virtual Network User Mode Linux (VNUML), a network virtualization environment based in User Mode Linux virtual machines running the DTN reference implementation. The simulation environment allows us to explore the applicability and behavior of the DTN architecture in real isolated-areas scenarios in developing countries. Furthermore, new developments built in the reference implementation (dynamic routing, multicast, anycast) can be easily debugged and analyzed.
\end{abstract}

\section{Categories and Subject Descriptors}

C.2 [Computer-Communication Networks]: Miscellaneous; C.0 [General]: Modeling of computer architecture

\section{General Terms}

Experimentation

\section{Keywords}

Simulation, Delay Tolerant Networks, Visualization tool

\section{INTRODUCTION}

In rural areas, mainly in developing countries, it is often not feasible to deploy the interactive and high capacity connectivity that the Internet allows specialists. However, it is easy to achieve a lower quality connectivity, often intermittent, for example, using solar powered energy hungry VHF or HF radios or wireless routers, or traditional and expensive modem phone calls. Sometimes the only way to get the data passed is using mobile nodes in buses, boats or other terrestrial vehicles, low earth orbiting (LEO) satellites, planes, etc. In these and other cases there is not a direct path between origin and destination. In addition, in this challenged networking environments the latency and available bandwidth between nodes can be substantially worse than those available in today's Internet. Approaches dealing somehow with this type of connectivity are the Saami Network Connectivity Project in Sweden [6], First Mile Solutions projects (DakNet [12], etc) and Wizzy Digital Courier Project [15] in South Africa.

Copyright is held by the author/owner(s). CHANTS'08, September 15, 2008, San Francisco, California, USA. ACM 978-1-60558-186-6/08/09.
Dealing with this intermittent, indirect, and non scheduled connectivity is the objective of the Delay Tolerant Networking (DTN) architecture [3], originally developed for deep space communications [2], but now also applied to terrestrial scenarios, including sensor networks and communication infrastructure for poorly connected regions [8].

This paper describes a simulation framework of the DTN architecture and explores the applicability of DTN to real scenarios found in projects of the Spanish-American Health Link Program (EHAS, in Spanish "Enlance Hispano Americano de Salud") [11]. It is structured as follows: section 2 introduces the concepts of the DTN architecture; section 3 presents the simulation environment for the DTN architecture and section 4 reviews our results and explores future work.

\section{DELAY TOLERANT NETWORKING}

The DTN architecture defines a store-and-forward message switched overlay that operates above the traditional transport layer (e.g. UDP and TCP) and may interconnect two or more dissimilar networks with arbitrary underlying protocol stacks. Unlike the traditional Internet architecture which requires end-to-end path between a source and destination, DTN can store messages (bundles) for long periods of time at intermediate nodes until the next hop is available.

Early versions of the DTN architecture included the concept of regions. Two nodes were in a same region if they could communicate between them without using a DTN gateway and sharing the same internetworking protocol. Bundles are passed from the source to destination via one or more gateways using tuples in the form (region $i d$, node id) for identified DTN nodes (e.g. bundles://AX25/host: //charupa).

In the current version of the architecture, DTN nodes are identified by a string called endpoint. Each Endpoint Identifier (EID) is expressed syntactically as a Uniform Resource Identifier (URI) [13]. The URI syntax has been designed as a way to express names or addresses for a wide range of purposes, and is therefore useful for constructing DTN names (e.g. dtn://central.dtn). Unlike the concept of region, which was tied more closely to assumptions of the network topology, endpoints are not required to be related to routing or topological organization. Such a relationship is not prohibited; moreover, in some environments using EID in this way may be advantageous.

In order to make an efficient use of the communication channel, DTN applications can transmit relatively large messages as the unit of transmission and acknowledgement. Tak- 


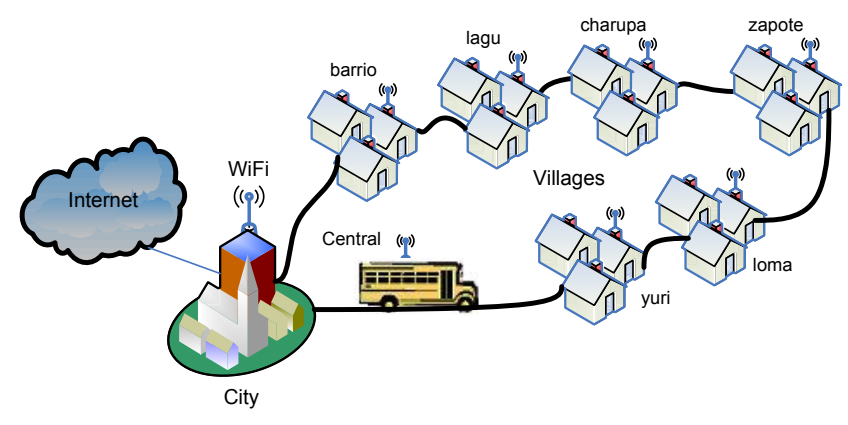

Figure 1: Rural network with a mobile wireless router in a bus.

ing advantage of this property, the messages can be physically sent end to end using a USB key, CD or DVD, through some "postal network" like in PostmanNet Project [14].

Another important aspect of the DTN is the use of temporal and scheduling information. The routing system may be able to pre-schedule the time at which messages should be sent, and even in case of two or more underlying networks (e.g. IP, LEO satellite networks, modem links, postal network, etc.), the path to send the bundles. Routing selection may also depend on other aspects like message source and destination, size and other factors. In fact, the routing is one of the most important aspects of the DTN development.

\section{DTN SIMULATION ENVIRONMENT}

The first tests with the DTN reference implementation were done using real equipment, but it did not allow us to create new and complex scenarios. In order to determine the applicability of the DTN concepts it is helpful to simulate the architecture and protocols in real scenarios.

We tried to validate the DTN concept with the DTN reference implementation [4] available using the following two scenarios: a mobile wireless router carried in a bus or other vehicle (boat, bicycle, etc.) circulates in rural areas, connecting endpoints while it is close to them, in the same way the DakNet Project does. (See Figure 1); and a typical setup deployed in EHAS projects. A hierarchical network in which health posts with an VHF radio connects its reference health center some times a day using a variant of the AX-25 packet radio protocol [1]. Health centers are connected by means of several short telephone calls, to a central server with Internet connection. (See Figure 2).

Therefore, we decided to use Virtual Network User Mode Linux (VNUML) [9] to create the simulation environment. VNUML is an open-source virtualization tool designed to easily define and test complex network simulation scenarios based on the User Mode Linux (UML) [5] virtualization software. With VNUML it is possible to test network applications and services over complex testbeds made of several nodes and networks inside one Linux machine. It is made of two main components: the VNUML language used to write down the specifications of the scenario, and a parser (writing in Perl) that interprets the language and builds and manages the simulation, hiding all UML complexity to the user.

Figure 3 illustrates the simulation scheme of a virtual network implemented to simulate the first real scenario in the host volatil. It was implemented with seven virtual machines, six of them simulate six villages (yuri, lagu, loma,

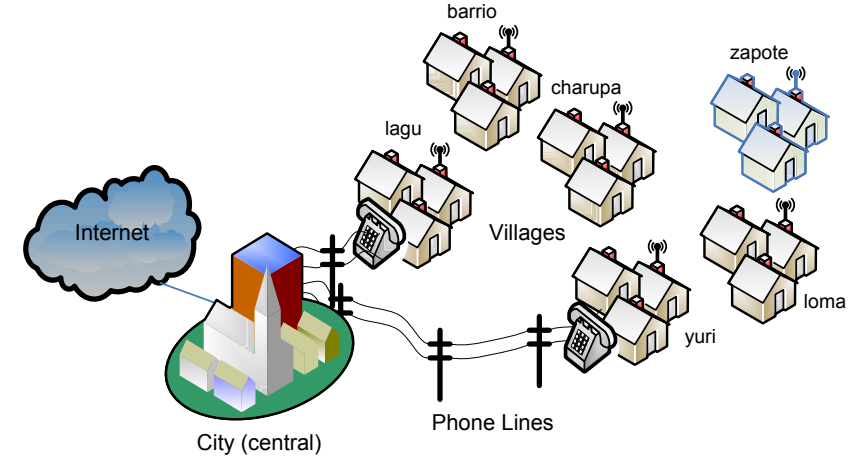

Figure 2: A part of the EHAS network in Alto Amazonas Province of Peru.

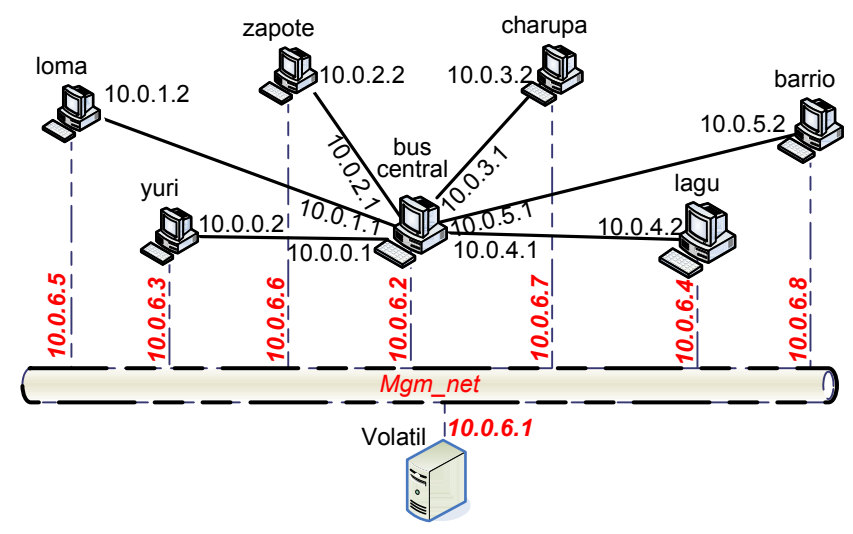

Figure 3: VNUML simulation scheme for a rural network with a mobile wireless router in a bus.

zapote, charupa and barrio), and the seventh (central) simulates the bus with the mobile wireless router. All the villages were connected by a point-to-point wireless link with the bus; this was simulated with the WiFi Layer for UML [10]. During the simulation, the virtual machine that simulated the bus (central) was only able to be connected to one village.

Figure 4 shows the simulation scheme of the virtual network implemented to simulate a part of the EHAS network in the same real machine. The connections by AX-25 between health posts in villages (loma, zapote, charupa and barrio) and health centers (yuri and lagu) were simulated setting the UML switch mode to PPP and fixing the bandwidth to 9600 bps. Health centers and the central server (central virtual machine) are connected by telephone calls using UUCP. It was simulated setting the UML switch mode to PPP, but in this case the bandwidth was fixed to 33.6 kbps.

The first simulations were made with the version of the DTN reference implementation that included the concept of DTN regions. In this case we simulated two variants of the rural network with the mobile wireless router in a bus. In the first one, the bus and the villages were in the same region. The villages basically have the same configuration: all have only one connection with the bus and in all them defined static routes to the remaining villages through the bus. In the second variant, every village and the bus were 


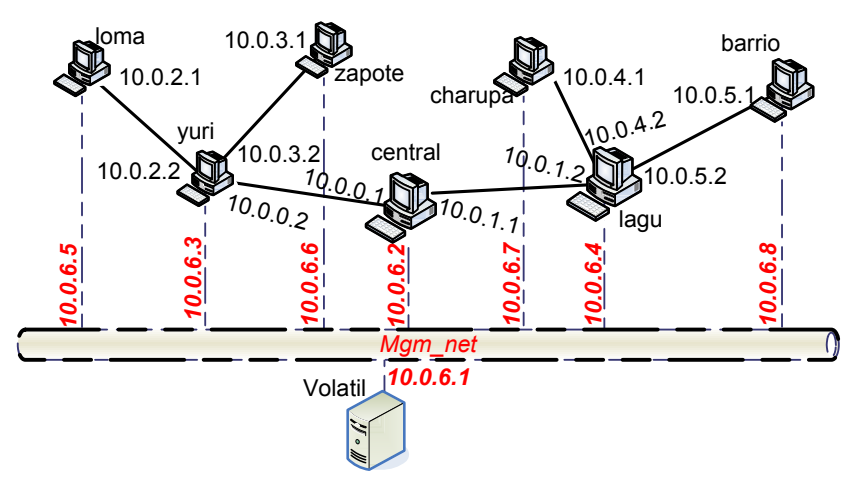

Figure 4: VNUML simulation scheme for the EHAS network.

located in its own region. The configuration of the villages is similar to the first variant: they only have connection with the bus, but in this case there are not any defined routes to all the nodes of the network, instead, routes were defined only to regions, independently of the number of nodes in a region.

In both cases the architecture worked perfectly, which seems to proof that the concept of region was ambiguous and depends on the network designer criteria. One important issue is what happens in case of a large number of regions. In fact, simultaneously with the analysis of this problem, the concept of region was eliminated in the new draft.

While the simulations were being performed was published, by the DTNRG group [7], the new version of the DTN reference implementation based on the new draft of the architecture. In this version the nodes are identified by an URI scheme like dtn://central.dtn.

Finally, the EHAS network in Alto Amazonas Province of Peru was simulated. In this case, because of the problem in the DTN reference implementation with the default route, all the villages in the network had static routes to the remaining villages through the DTN router to which they are connected.

\section{CONCLUSIONS AND FUTURE WORK}

The simulation environment with virtual machines proved to be a valuable tool for the visualization of DTN based architectures, like those easy to deploy in disadvantaged areas of developing countries. It is also useful for debugging and performance evaluation of DTN protocols and applications based on real software implementations. The DTN architecture and protocols are still in draft state, and the reference implementation follows the drafts as close as possible; this simulation environment may help to speed up the development of the standards.

Further work to make the simulation environment more useful might be to extend the VNUML language to cleanly express the connection and disconection patterns, as well as with data gathering tools, so that the behaviour of the network can easily be tracked.

\section{ACKNOWLEDGMENTS}

This work was carried out in the Applications of Delay Tolerant Networking in isolated areas in developing countries project funded by Universidad Politécnica de Madrid.

\section{REFERENCES}

[1] W. Beech, D. Nielsen, and J. Taylor. AX.25 link access protocol for amateur packet radio v2.2. Technical report, Tucson Amateur Radio Corporation, 1997.

[2] S. Burleigh, V. Cerf, R. Durst, K. Fall, A. Hooke, K. Scott, and H. Weiss. The Interplanetary Internet: A Communications Infrastructure for Mars Exploration. In 53rd International Astronautical Congress, The Second World Space Congress, Houston, Texas, USA, 2002.

[3] V. G. Cerf, S. C. Burleigh, A. J. Hooke, L. Torgerson, R. C. Durst, K. L. Scott, K. Fall, and H. S. Weiss. Delay-tolerant network architecture. Internet Draft, (draft-irtf-dtnrg-arch-03.txt), July 2005.

[4] M. Demmer, E. Brewer, K. Fall, S. Jain, M. Ho, and R. Patra. Implementing delay tolerant networking. Technical report, Intel Research, 2004.

[5] J. Dike. User-mode linux. In Proceedings of the 5th Annual Linux Showcase and Conference, pages 27-34, Oakland, Nov. 2001.

[6] A. Doria, M. Uden, and D. P. Pandey. Providing connectivity to the saami nomadic community. In Proceedings of the Second International Conference on Open Collaborative Design for Sustainable Innovation, 2002.

[7] Delay-Tolerant Networking Research Group (DTNRG) web site. http://www.dtnrg.org.

[8] K. Fall. A delay-tolerant network architecture for challenged internets. In Proceedings of the 2003 Conference on Applications, technologies, architectures, and protocols for computer communications (SIGCOMM), pages 27-34. ACM Press, 2003.

[9] F. Galán, D. Fernández, J. Ruiz, O. Walid, and T. de Miguel. A virtualization tool in computer network laboratories. In Proceedings of the 5th International Conference on Information Technology Based Higher Education and Training (ITHET'04), pages 27-34, Istambul, May 2004.

[10] V. Guffens, G. Bastin, and O. Bonaventure. An emulation infrastructure for multi-hop wireless communication networks. Internal report, CESAME, Université Catholique de Louvain, 2004.

[11] A. Martínez, V. Villarroel, J. Seoane, and F. del Pozo. Rural Telemedicine for Primary Healthcare in Developing Countries. IEEE Technology 85 Society Magazine, 23(2):13-22, 2004.

[12] A. S. Pentland, R. Fletcher, and A. Hasson. Daknet: Rethinking connectivity in developing nations. IEEE Computer, 37(1), Jan. 2004.

[13] K. Scott and S. Burleigh. Bundle protocol specification. Internet Draft, (draft-irtf-dtnrg-bundle-spec-03.txt), July 2005.

[14] R. Y. Wang, S. Sobti, N. Garg, E. Ziskind, J. Lai, and A. Krishnamurthy. Turning the postal system into a generic digital communication mechanism. SIGCOMM Comput. Commun. Rev., 34(4):159-166, 2004.

[15] Wizzy Digital Courier home page. http://www.wizzy.org.za. 